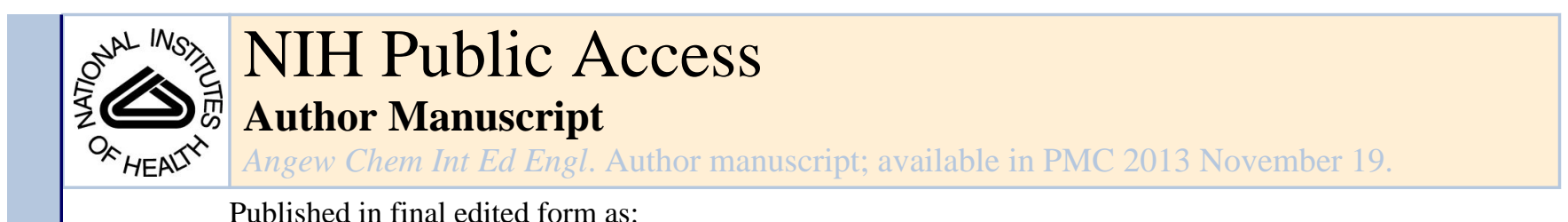

Published in final edited form as:

Angew Chem Int Ed Engl. 2012 November 19; 51(47): 11899-11903. doi:10.1002/anie.201206595.

\title{
A Bioreducible Polymer for Efficient Delivery of Fas-Silencing siRNA into Stem Cell Spheroids and Enhanced Therapeutic Angiogenesis*
}

\author{
Dr. Min Suk Shim ${ }^{[\dagger],[\ddagger],}$ \\ Department of Biomedical Engineering, Washington University, St. Louis, MO 63130 (USA) \\ Dr. Suk Ho Bhang[t],[‡], \\ Department of Biomedical Engineering, Washington University, St. Louis, MO 63130 (USA) \\ Dr. Kyunghwan Yoon, \\ Department of Biomedical Engineering, Washington University, St. Louis, MO 63130 (USA)
}

Prof. Kyunghee Choi, and

Department of Pathology and Immunology, Washington University School of Medicine, St. Louis, MO 63110 (USA)

Prof. Younan Xia*

Department of Biomedical Engineering, Washington University, St. Louis, MO 63130 (USA), Tel: $+1-404-385-3209$

Kyunghwan Yoon: younan.xia@bme.gatech.edu

\begin{abstract}
This paper describes a bioreducible polymer for efficient delivery of Fas-silencing small interfering RNA (Fas siRNA) into human mesenchymal stem cells (hMSCs). The genetically modified hMSCs could be formulated as spheroids with enlarged sizes ( $\geq 800 \mu \mathrm{m}$ in diameter) to enhance their angiogenic efficacy. The Fas siRNA delivered into the cytoplasm of hMSCs via the bioreducible polymer could efficiently inhibit Fas expression, leading to effective inhibition of hypoxia-induced apoptosis in the core of an enlarged spheroid of hMSCs. The enlarged spheroid not only increased the number of viable hMSCs but also significantly enhanced the secretion of bioactive angiogenic growth factors per hMSC relative to small spheroids that were not treated with Fas siRNA. Twenty-eight days after transplantation into mouse with ischemic hindlimbs, the enlarged hMSC spheroids significantly enhanced cell survival, limb salvage, blood perfusion, and vessel formation while substantially reducing muscle degeneration and tissue fibrosis. This study demonstrates a promising strategy that combines siRNA and spheroid-based stem cell therapies using a stimuli-responsive nonviral siRNA carrier for the efficient treatment of ischemic disease.
\end{abstract}

\footnotetext{
** This work was supported in part by an NIH Director's Pioneer Award (DP1 OD000798 to Y.X.) and NIH grants from the National Heart, Lung and Blood Institute (NHLBI, R01 HL55337 and R01 HL63736 to K.C.). The authors thank Dennis Oakley of the Bakewell Neuroimaging Core at Washington University School of Medicine for assistance with the confocal microscopy imaging.

Correspondence to: Younan Xia.

$[\dagger]$ These two authors contributed equally to this work.

$[\ddagger]$ Current address: The Wallace H. Coulter Department of Biomedical Engineering, Georgia Institute of Technology and Emory University; School of Chemistry \& Biochemistry and School of Chemical \& Biomolecular Engineering, Georgia Institute of

Technology, Atlanta, GA 30332
} 


\title{
Keywords
}

Angiogenesis; Cell spheroids; Human mesenchymal stem cell; Nanoparticles; RNA interference

\begin{abstract}
Adult mesenchymal stem cells (MSCs) hold great potential for the treatment of ischemic disease because they can contribute to angiogenesis by secreting a broad spectrum of angiogenic growth factors. ${ }^{[1]}$ However, the low survival rate and poor engraftment of the transplanted MSCs limit the use of MSC-based therapy in a clinical setting. ${ }^{[2]}$ Recently, it was shown that transplanting stem cells as spheroids of $c a .200 \mu \mathrm{m}$ in diameter could be a promising strategy for improving their angiogenic efficacy. ${ }^{[3]}$ The mild hypoxic condition established in the core of a spheroid (arising from the limited diffusion of nutrients and oxygen) can precondition the cells to an ischemic environment. ${ }^{[4]}$ Through this natural preconditioning, the cells can acquire resistance to the hypoxic condition by increasing the production of various growth factors that are necessary for survival. ${ }^{[4,5]}$ However, for MSCbased spheroids, the therapeutic efficacy of this approach is limited by the number of MSCs that could be included in the spheroid. When the spheroid was increased beyond a certain size, a large number of MSCs in the core underwent severe hypoxia establishment and eventually died (unpublished observations). As a result, there is a strong need to develop an effective method for increasing the survival rate of MSCs in a large spheroid to enhance their therapeutic efficacy.
\end{abstract}

One way to improve the survival rate of MSCs in a spheroid is to inhibit their apoptosis, which is activated by interactions between Fas and its ligand (FasL) ${ }^{[6]}$ It was reported that inhibition of Fas expression could protect the cells from apoptosis. ${ }^{[7]}$ Based on this notion, we hypothesized that hypoxia-induced apoptosis in a spheroid could be mitigated by inhibiting Fas expression through the delivery of Fas-silencing small interfering RNA (Fas siRNA), resulting in successful formulation of spheroids with enlarged sizes. Due to the increased number of viable MSCs and thereby augmented total secretion of paracrine angiogenic factors, the enlarged spheroids were anticipated to significantly enhance angiogenesis. In recent years, various cationic polymers have been actively explored as efficient nonviral vectors because they can strongly condense negatively charged siRNAs and protect them from degradation by nuclease. ${ }^{[8]}$ However, the use of a high molecular weight cationic polymer such as polyethylenimine (PEI, a commercially available transfecting agent) is problematic due to its non-biodegradability and thus high cytotoxicity. ${ }^{[9]}$ As a result, stimuli-responsive, biodegradable polymers have recently emerged as effective nonviral carriers for reducing cytotoxicity and thus enhancing transfection efficiency by taking advantage of cellular stimuli-responsive degradation and efficient release of nucleic acids. ${ }^{[10]}$

In this study, branched poly(disulfide amine) (B-PDA), a bioreducible cationic polymer containing disulfide bonds in the backbone, was developed for efficient intracellular delivery of Fas siRNA into human MSCs (hMSCs). The salient features of this polymer include: $i$ ) efficient complexation of flexible cationic amino branches with Fas siRNA; ii) high proton buffering capacity for efficient endosomal escape because the cationic branches are consisted of primary, secondary, and tertiary amines with a broad range of pKa values, 
which represents a major advantage as compared to the previously reported bioreducible polymers; ${ }^{[10 \mathrm{c}, \mathrm{d}]}$ iii) facilitated release of Fas siRNA in the cytoplasm - an intracellular target site for RNA interference (RNAi), due to rapid degradation of disulfide bonds in the reductive environment; and $i v$ ) low toxicity and efficient body clearance owing to efficient biodegradation. As shown in Figure 1a, bioreducible B-PDA can be used to complex with Fas siRNA and release it in the cytoplasm of a hMSC. The Fas-silenced hMSCs can then be formulated as enlarged spheroids and transplanted into mouse ischemic hindlimbs as paracrine secretors for therapeutic angiogenesis.

The synthesis of B-PDA involved polymerization of a Boc-protected oligoamine monomer and disulfide-bearing $N, N^{\prime}$-cystaminebisacrylamide via Michael addition conjugation, followed by deprotection of the Boc groups (see Figure S1 in the Supporting Information for details). As determined by gel permeation chromatography (GPC), the B-PDA had molecular weights of $\mathrm{M}_{\mathrm{n}} \approx 6.0 \mathrm{kDa}$ and $\mathrm{M}_{\mathrm{w}} \approx 7.4 \mathrm{kDa}$, together with a narrow polydispersity index of 1.23 . The B-PDA exhibited high proton buffering capacity comparable to branched PEI as demonstrated by the titration curves in Figure $\mathrm{S} 2$. This result implies that the Fas siRNA/B-PDA polyplexes should be able to escape from the acidic endosome via the hypothetical "proton buffering effect" after they have entered cells. ${ }^{[11]}$

The redox-sensitive degradation of B-PDA was investigated by monitoring its molecular weights after incubation in a $10 \mathrm{mM}$ dithiothreitol (DTT) solution for different periods of time. Our results indicate that the B-PDA could be completely degraded into short segments with $\mathrm{M}_{\mathrm{w}}$ less than $600 \mathrm{Da}$ within $3 \mathrm{~h}$ under the reductive condition (Figure S3a). The multiple amino groups on the flexible branches of B-PDA could efficiently complex with negatively charged Fas siRNA, forming stable polyplexes with varying amine to phosphate (N/P) ratios. As shown by TEM images (Figure 1b, left), compact Fas siRNA/B-PDA polyplexes with diameters in the range of 60-80 nm were formed at an N/P ratio of 100 . After incubation in reductive DTT solution, the Fas siRNA/B-PDA polyplexes became dramatically loosen (Figure 1b, right). The destabilized Fas siRNA/B-PDA polyplexes after reduction was also confirmed by dynamic light scattering analysis (Figure S3b). Gel electrophoresis assay also demonstrated the redox-triggered disassembly of the Fas siRNA/B-PDA polyplexes (Figure 1c). It is well-known that intracellular localization and disassembly of Fas siRNA from a carrier determine the efficiency of RNAi. ${ }^{[12]}$ For the Fas siRNA/B-PDA polyplexes, we confirmed their efficient disassembly inside hMSCs by confocal laser scanning microscopy. As represented by many red dots in the confocal micrograph (Figure S3c), a significant amount of free siRNA (labeled with Cy3 dye) was released from the Alexa Fluor 488-labeled polymer chains (green dots). This result confirms that the redox-sensitive degradation of disulfide bonds in B-PDA facilitated the cytoplasmic release of Fas siRNA.

The Fas silencing efficiencies of various types of Fas siRNA/polymer polyplexes or naked siRNA were determined by incubating them with hMSCs for $48 \mathrm{~h}$. For Fas siRNA/B-PDA polyplexes, an N/P ratio of 100 was used, which was optimized by considering their Fas silencing efficiency and cytotoxicity. Branched PEI (25 kDa, N/P ratio of 10) was used as a non-reducible control. The transfected hMSCs were cultured as spheroids under hypoxic condition (e.g., $1 \%$ oxygen and serum-free medium) using a hanging drop method (see 
Supporting Information for details) to mimic the in vivo ischemic environment. As shown in Figure $2 \mathrm{a}$ and $\mathrm{b}$, the level of Fas messenger RNA (Fas mRNA) was substantially suppressed by the Fas siRNA/B-PDA polyplexes. Importantly, the gene silencing efficiency of Fas siRNA/B-PDA polyplexes was about 1.5-fold higher than that of Fas siRNA/PEI polyplexes (Figure 2b). It is known that highly stable complexation of siRNA with nondegradable PEI limits the dissociation of siRNA from the PEI polyplexes. ${ }^{[13]}$ Therefore, the more efficient inhibition of Fas expression by the B-PDA relative to PEI can be attributed to its capability to facilitate the cytoplasmic release of Fas siRNA (Figure S3c).

The effect of Fas inhibition by Fas siRNA/B-PDA polyplexes on the apoptosis of hMSCs was investigated by quantifying mRNA expressions of anti-apoptotic Bcl-XL and proapoptotic Bax (Figure 2, a, c, d). Efficient inhibition of Fas expression by siRNA/B-PDA polyplexes led to concomitantly increased Bcl-XL expression and decreased Bax expression, demonstrating significantly down-regulated Fas-inductive apoptosis gene cascades. In addition, Fas siRNA/B-PDA polyplexes significantly down-regulated caspase-3, a protease involved in Fas-mediated apoptosis (Figure S4, a and b). ${ }^{[14]}$ As a result, treatment with Fas siRNA/B-PDA polyplexes greatly increased the viability of hMSCs in the spheroids as compared to other groups (Figure 2e and Figure S4, $\mathrm{c}$ and d). The substantially inhibited apoptosis of hMSCs by Fas siRNA/B-PDA polyplexes also affected the morphologies of their spheroids. As shown by SEM images (Figure $2 \mathrm{f}$ and Figure S5), hMSCs treated with Fas siRNA/B-PDA polyplexes were successfully formulated as enlarged spheroids due to their reinforced anti-apoptotic activity even under severe hypoxic conditions in the spheroid core. A majority of the enlarged spheroids had diameters in the range of $800-1000 \mu \mathrm{m}$. Although hMSCs treated with Fas siRNA/PEI polyplexes could also be cultured as enlarged spheroids with similar diameters, most of them showed apoptotic core deformation. The spheroids formed with hMSCs without any treatment or treated with naked Fas siRNA easily dissociated into individual cells due to severe hypoxic conditions caused by both inner and outer microenvironments.

The enlarged hMSC spheroids treated with Fas siRNA/B-PDA polyplexes significantly increased the expression of hypoxia-induced survival factor (i.e., HIF-1a) and antiapoptotic, angiogenic growth factors, including vascular endothelial growth factor (VEGF), fibroblast growth factor 2 (FGF2), and hepatocyte growth factor (HGF) (Figure S6, a-f). HIF-1a has been known to stimulate the production of angiogenic growth factors, as well as to trigger anti-apoptotic signaling cascades. ${ }^{[15]}$ Therefore, the increased secretion of VEGF, FGF2, and HGF in the hMSC spheroids treated with Fas siRNA/B-PDA polyplexes seems to be related to the increased expression of HIF-1a. It is worth noting that the amount of secreted angiogenic factors per individual hMSC for enlarged hMSC spheroids treated with Fas siRNA/B-PDA polyplexes was much higher (about 2-fold higher) than that of small hMSC spheroids (diameters ranging from 250 to $350 \mu \mathrm{m}$ ) that were not treated with Fas siRNA (Figure 2 $\mathrm{g}$ and Figure S6, $\mathrm{g}$ and $\mathrm{h}$ ). The enhanced paracrine secretion of enlarged hMSC spheroids can be attributed to the substantial increase in HIF-1a expression due to their exposure to greater hypoxic conditions compared to the small counterparts.

To determine if the enhanced viability of hMSCs in the enlarged spheroid with Fas siRNA transfection [enlarged spheroid (+siRNA)] and their increased secretion of paracrine 
angiogenic factors can stimulate therapeutic angiogenesis, we evaluated their in vivo angiogenic efficacy in a mouse model of hindlimb ischemia. For comparison, we also prepared and transplanted the following samples: enlarged hMSC spheroids formulated with the same initial number of hMSCs without Fas siRNA transfection [enlarged spheroid (-siRNA)], Fas siRNA-transfected hMSC spheroids with a small number of hMSCs [small spheroid (+siRNA)], and small hMSC spheroids without Fas siRNA transfection [small spheroid (-siRNA)]. The reason for including small spheroid (+siRNA) and small spheroid (-siRNA) was to evaluate the effects of the number of hMSCs and the Fas siRNA-mediated anti-apoptosis on the spheroid's angiogenic efficacy, respectively. It should be noted that the enlarged spheroid (-siRNA) could not maintain as spheroids due to severe hypoxia-induced apoptosis. Prior to in vivo study, the bioactivity of growth factors secreted from the enlarged spheroid (+siRNA) was confirmed by the significantly reduced apoptosis of human umbilical vein endothelial cells (HUVECs) cultured in the conditioned medium obtained from the enlarged spheroid (+siRNA) (see Figure S7 for details). The sustained retention of transplanted hMSCs as paracrine secretors is critical to their effectiveness in angiogenesis. Anti-apoptosis and cell survival of transplanted hMSC spheroids in mouse ischemic tissues were evaluated by double immunofluorescent staining of caspase- 3 and anti-human nuclear antigen (HNA) in the tissues. When enlarged spheroid (+siRNA) was transplanted, a significantly larger number of hMSCs survived in the ischemic tissues compared to other types of spheroids (Figure S8). Interestingly, there was no statistical difference in antiapoptosis and cell survival of hMSCs between small spheroid (+siRNA) and small spheroid (-siRNA) groups (Figure S8, b and c). This finding implies that Fas siRNA treatment is not effective for small hMSC spheroids because the survival of a small number of hMSCs can be effectively maintained by forming spheroids.

We also evaluated the therapeutic angiogenic efficacies of various types of hMSC spheroids in mouse ischemic hindlimbs by their physiological status, laser Doppler perfusion imaging, and histological analysis (Figure 3). As shown in Figure 3, a and c, the limb muscles of mice treated with enlarged spheroid (+siRNA) were effectively protected against ischemic damage, whereas all of the untreated mice with ischemic hindlimbs showed rapid limb necrosis and complete limb loss via autoamputation by day 28 . The mice treated with enlarged spheroid (+siRNA) underwent a significantly reduced limb loss rate (ca. 20\%) in comparison with other groups. Laser Doppler perfusion imaging revealed that the blood perfusion in the ischemic limbs treated with enlarged spheroid (+siRNA) was significantly improved compared to other groups (Figure 3, b and d). Histological analysis also revealed that the ischemic limbs treated with enlarged spheroid (+siRNA) showed substantially reduced tissue degeneration and fibrosis when compared with other groups (Figure 3, e and f, and Figure S9).

The transplantation of enlarged spheroid (+siRNA) significantly improved the formation of both arterioles and capillaries when compared to other types of hMSC spheroids (Figure 4). The transplantation of enlarged spheroid (+siRNA) also induced the formation of new microvessels most efficiently compared to other groups, as evident from a significantly increased number of BrdU-positive cells (Figure 4d). In addition, expressions of intercellular adhesion molecule (ICAM) and vascular cell adhesion molecule (VCAM) were the highest 
for the ischemic tissues treated with enlarged spheroid (+siRNA) (Figure S10a). Enhanced secretion of paracrine factors from the enlarged spheroid (+siRNA) might have activated the host cells to express more adhesion molecules. The increase in ICAM and VCAM expressions for the activated host cells might promote the recruitment of endothelial progenitor cells to ischemic sites and contribute to neovascularization.

To validate our hypothesis that the enhanced angiogenic efficiency of enlarged hMSC spheroids is closely correlated with their increased secretion of angiogenic and antiapoptotic growth factors, expressions of human VEGF (hVEGF) and human FGF2 (hFGF2) secreted from various hMSC spheroids were assessed 28 days after transplantation (Figure S10b). The highest levels of hVEGF and hFGF2 expressions were observed in mice that received enlarged spheroid (+siRNA), whereas hVEGF and hFGF2 expressions were not detected in mice transplanted with enlarged spheroid (-siRNA) (Figure S10b). We also quantified the expressions of human specific SM-a actin and CD31 to evaluate if microvessels were directly derived from hMSCs. Importantly, we did not observe noticeable expressions of human specific SM-a actin and CD31, confirming that the enhanced angiogenesis was mainly caused by the increased secretion of paracrine angiogenic factors from the enlarged hMSC spheroids (Figure S10b).

In summary, we have shown that Fas siRNA delivery into hMSCs via bioreducible B-PDA successfully suppressed Fas expression and thus led to effective inhibition of hypoxiainduced apoptosis in the enlarged hMSC spheroids (Figure S11). The enlarged hMSC spheroids significantly enhanced angiogenesis in mouse ischemic hindlimbs. This study demonstrates that an interdisciplinary approach of integrating sequence-specific RNAi, nonviral gene delivery, and spheroid-based stem cell delivery may offer a powerful therapeutic tool for the efficient treatment of ischemic disease.

\section{Supplementary Material}

Refer to Web version on PubMed Central for supplementary material.

\section{References}

1. a) Bianco P, Riminucci M, Gronthos S, Robey PG. Stem Cells. 2001; 19:180-192. [PubMed: 11359943] b) Caplan AI, Dennis JE. J Cell Biochem. 2006; 98:1076-1084. [PubMed: 16619257] c) Kinnaird T, Stabile E, Burnett MS, Lee CW, Barr S, Fuchs S, Epstein SE. Circ Res. 2004; 94:678685. [PubMed: 14739163]

2. Tateishi-Yuyama E, Matsubara H, Murohara T, Ikeda U, Shintani S, Masaki H, Amano K, Kishimoto Y, Yoshimoto K, Akashi H, Shimada K, Iwasaka T. Lancet. 2002; 360:427-435. [PubMed: 12241713]

3. Bhang SH, Cho SW, La WG, Lee TJ, Yang HS, Sun AY, Baek SH, Rhie JW, Kim BS. Biomaterials. 2011; 32:2734-2747. [PubMed: 21262528]

4. Korff T, Kimmina S, Martiny-Baron G, Augustin HG. FASEB J. 2001; 374:447-457. [PubMed: 11156960]

5. Gaedtke L, Thoenes L, Culmsee C, Mayer B, Wagner E. J Proteome Res. 2007; 6:4111-4118. [PubMed: 17918984]

6. a) Song E, Lee SK, Wang J, Ince N, Ouyang N, Min J, Chen J, Shankar P, Lieberman J. Nat Med. 2003; 9:347-351. [PubMed: 12579197] b) Suarez-Pinzon W, Sorensen O, Bleackley RC, Elliott JF, Rajotte RV, Rabinovitch A. Diabetes. 1999; 48:21-28. [PubMed: 9892218] c) Kagi D, Vignaux F, 
Ledermann B, Burki K, Depraetere V, Nagata S, Hengartner H, Golstein P. Science. 1994; 265:528-530. [PubMed: 7518614]

7. Hamar P, Song E, Kökény G, Chen A, Ouyang N, Lieberman J. Proc Natl Acad Sci USA. 2004; 101:14883-14888. [PubMed: 15466709]

8. a) Gabrielson NP, Lu H, Yin L, Li D, Wang F, Cheng J. Angew Chem Int Ed. 2012; 51:11431147.b) Park TG, Jeong JH, Kim SW. Adv Drug Deliv Rev. 2006; 58:467-486. [PubMed: 16781003]

9. a) Fischer D, Bieber T, Li Y, Elsässer H, Kissel T. Pharm Res. 1999; 16:1273-1279. [PubMed: 10468031] b) Knauf MJ, Bell DP, Hirtzer P, Luo ZP, Young JD, Katre NV. J Biol Chem. 1988; 263:15064-15070. [PubMed: 3049599]

10. a) Lynn DM, Langer R. J Am Chem Soc. 2000; 122:10761-10768.b) Lin YL, Jiang G, Birrell LK, El-Sayed MEH. Biomaterials. 2010; 31:7150-7166. [PubMed: 20579726] c) Christensen LV, Chang CW, Kim WJ, Kim SW. Bioconjugate Chem. 2006; 17:1233-1240.d) Burke RS, Pun SH. Bioconjugate Chem. 2010; 21:140-150.

11. Boussif O, Lezoualc'h F, Zanta MA, Mergny MD, Scherman D, Demeneix B, Behr JP. Proc Natl Acad Sci U S A. 1995; 92:7297-7301. [PubMed: 7638184]

12. Detzer A, Overhoff M, Wünsche W, Rompf M, Turner JJ, Ivanova GD, Gait MJ, Sczakiel G. RNA. 2009; 15:627-636. [PubMed: 19228587]

13. Breunig M, Hozsa C, Lungwitz U, Watanabe K, Umeda I, Kato H, Goepferich A. J Control Release. 2008; 130:57-63. [PubMed: 18599144]

14. Nicholson DW, Ali A, Thornberry NA, Vaillancourt JP, Ding CK, Gallant M, Gareau Y, Griffin PR, Labelle M, Lazebnik YA, Munday NA, Raju SM, Smulson ME, Yamin TT, Yu VL, Miller DK. Nature. 1995; 376:37-43. [PubMed: 7596430]

15. a) Bernaudin M, Nedelec AS, Divoux D, MacKenzie ET, Petit E, Schumann-Bard P. J Cereb Blood Flow Metab. 2002; 4:393-403. [PubMed: 11919510] b) Ma D, Lim T, Xu J, Tang H, Wan Y, Zhao H, Hossain M, Maxwell PH, Maze M. J Am Soc Nephrol. 2009; 20:713-720. [PubMed: 19144758] 
a) Branched poly(disulfide amine) (B-PDA)
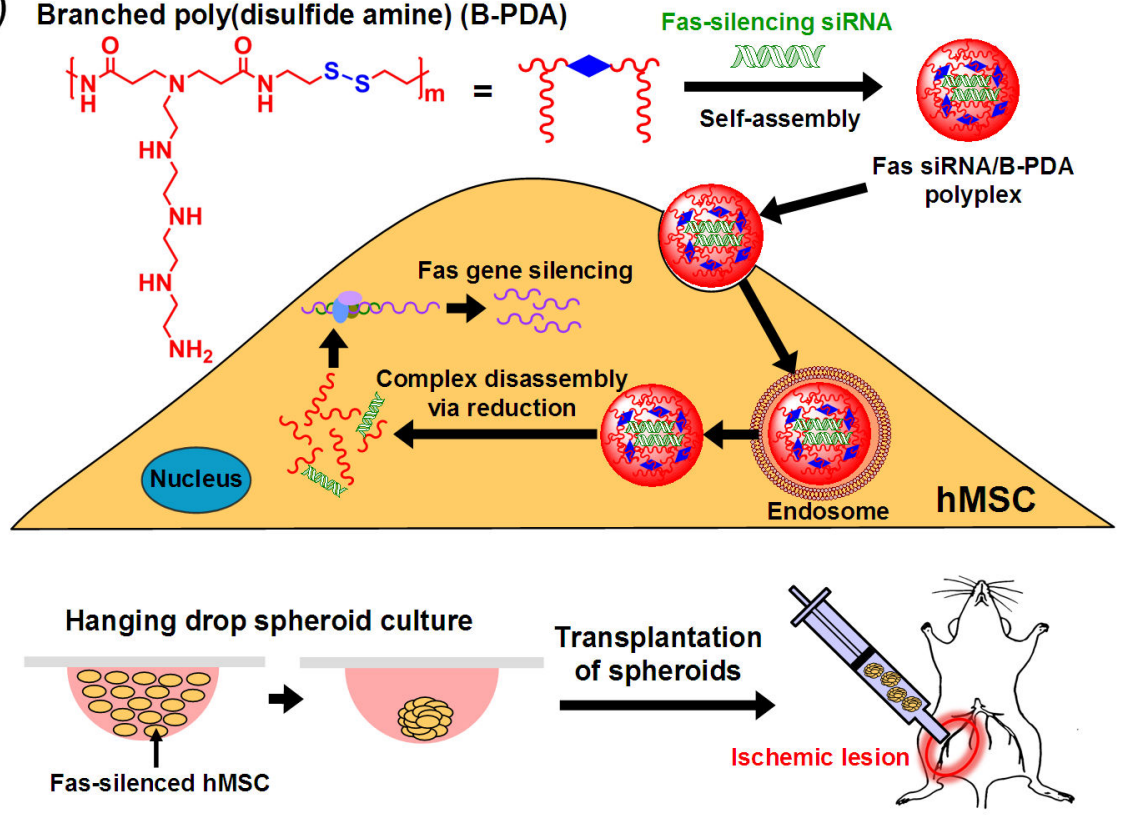

b) Before reduction
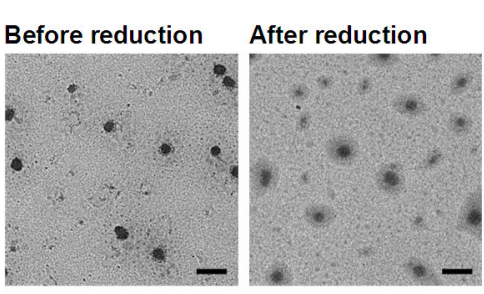

C)

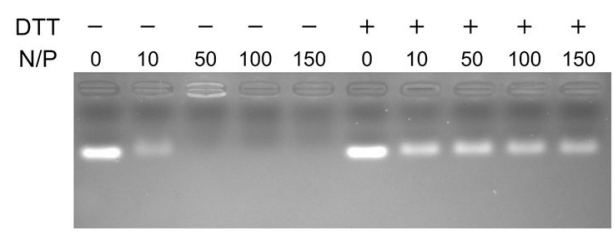

Figure 1.

(a) Intracellular trafficking of Fas siRNA/B-PDA polyplexes for the delivery of Fas siRNA into hMSCs and formation of enlarged spheroids of Fas-silenced hMSCs for the ischemia treatment. (b) TEM images of siRNA/B-PDA polyplexes before and after reduction at $37^{\circ} \mathrm{C}$ for $8 \mathrm{~h}$ in the presence of DTT $(10 \mathrm{mM})$. The scale bars correspond to $200 \mathrm{~nm}$. (c) Gel retardation assay of siRNA/B-PDA polyplexes obtained under varying N/P ratios before and after incubation with DTT $(10 \mathrm{mM})$ at $37^{\circ} \mathrm{C}$ for $4 \mathrm{~h}$. 
a)

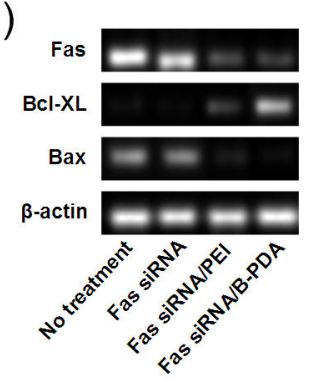

e)

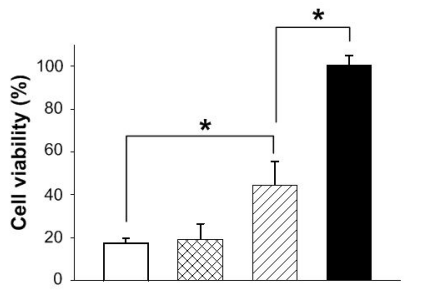

b)

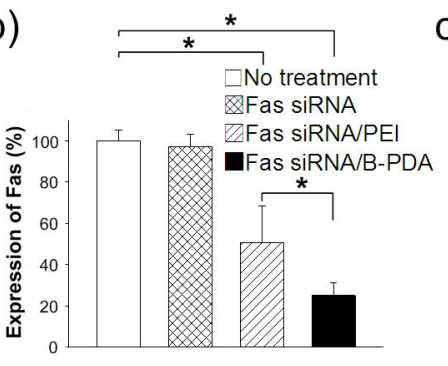

f) c)

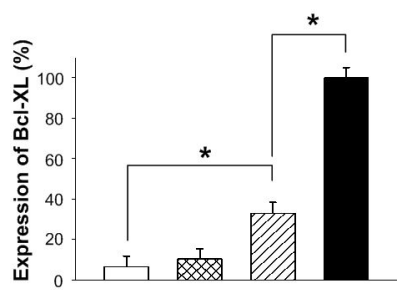

d)

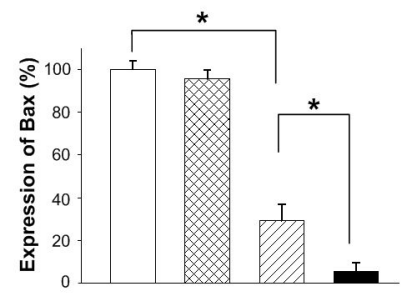

\section{Figure 2.}

(a-d) Anti-apoptotic activity of the enlarged hMSC spheroids treated with Fas siRNA via different delivery methods, as determined by (a) reverse transcription-polymerase chain reaction (RT-PCR) and (b-d) quantitative PCR (qPCR), respectively. (e) Cell viability of hMSC spheroids treated with Fas siRNA via different delivery methods, as determined by neutral red assay. The results are expressed as percentages relative to the Fas siRNA/B-PDA group. (f) SEM images of hMSC spheroids treated with Fas siRNA via different delivery methods under hypoxic culture. Scale bars correspond to $200 \mu \mathrm{m}$. (g) Quantification of VEGF secreted from hMSC spheroids after treatment with various Fas siRNA delivery methods, as quantified by enzyme-linked immunosorbent assay (ELISA). The hMSC spheroids were cultured under hypoxic conditions for 5 days. * denotes $p<0.05$. 
a)

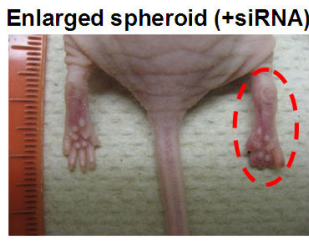

b)

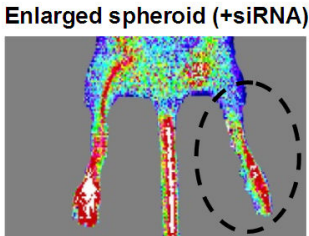

c)

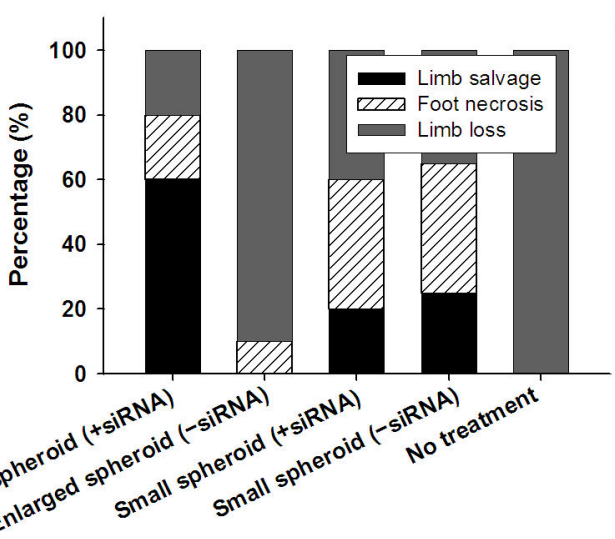

e)

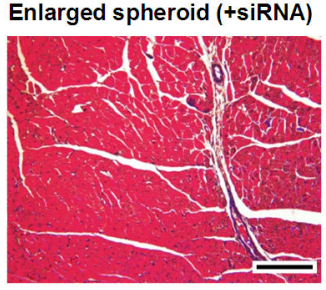

Small spheroid (-siRNA)

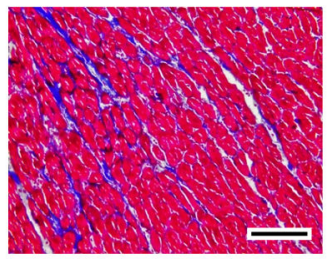

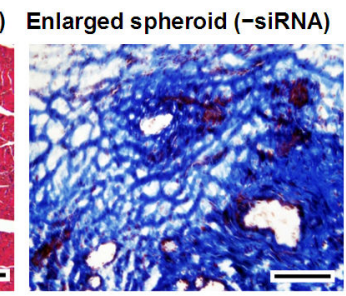

No treatment

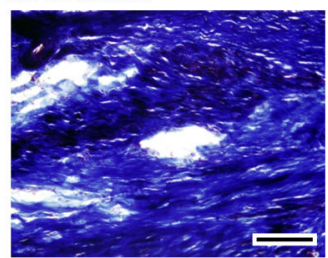

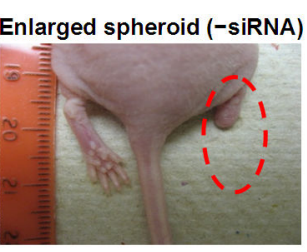
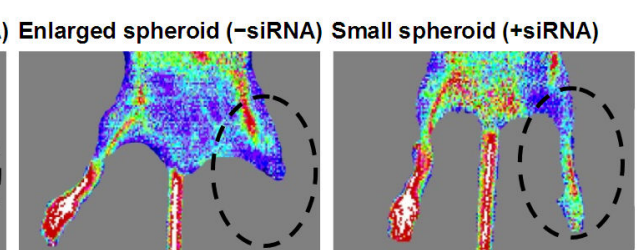

d)

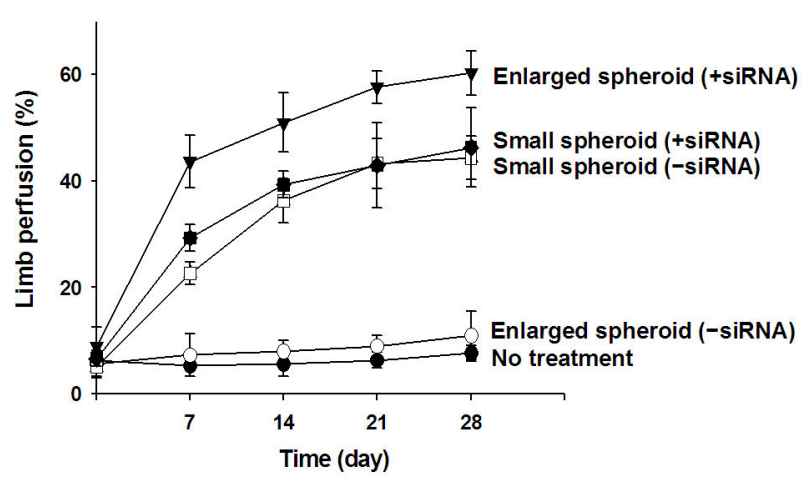

Small spheroid (-siRNA)

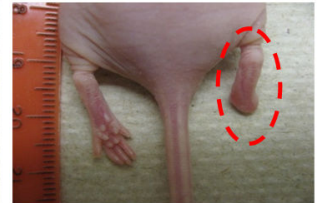

Small spheroid (-siRNA)

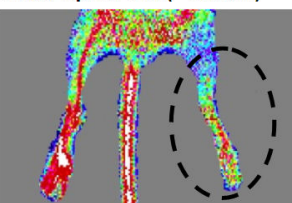

No treatment

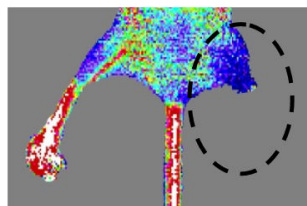

No treatment

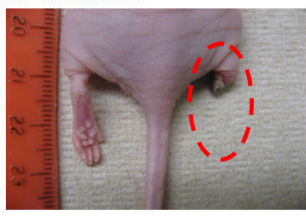

Figure 3.

Improvements of ischemic hindlimb salvage 28 days post transplantation of various types of hMSC spheroids. (a) Representative photographs and (b) laser Doppler perfusion images of the ischemic hindlimbs 28 days post treatment. Dashed eclipses indicate ischemic hindlimbs. (c) Physiological status of ischemic limbs 28 days post treatment. (d) Blood perfusion ratio of ischemic limbs measured by laser Doppler imaging 0,7,14,21, and 28 days post treatment. The ratio of blood perfusion between ischemic and normal limbs was significantly improved by the enlarged spheroid (+siRNA) at all time points. (e) Masson's trichrome staining $(\times 100)$ of ischemic tissues (red: normal muscle tissues; blue: fibrotic 
tissues) and (f) evaluation of fibrotic tissue area in the ischemic tissues 28 days post treatment. It should be noted that the enlarged spheroid (-siRNA) could not remain as spheroids due to intolerable hypoxia-induced apoptosis. The scale bars correspond to $50 \mu \mathrm{m}$. All photographs had the same magnification. * denotes $p<0.05$. 
a) Enlarged spheroid (+siRNA) Enlarged spheroid (-siRNA) Small spheroid (+siRNA)

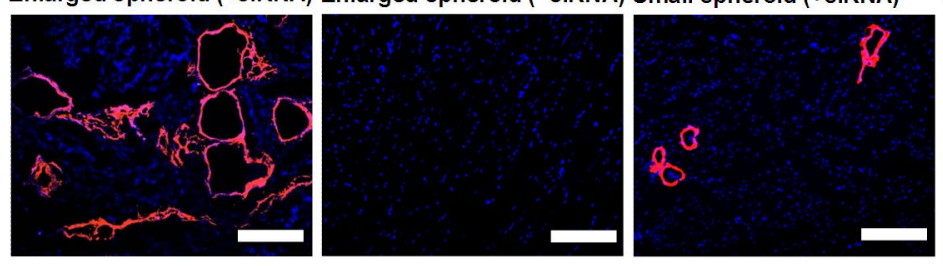

Small spheroid (-siRNA) No treatment

b) Enlarged spheroid (+siRNA) Enlarged spheroid (-siRNA) Small spheroid (+siRNA)

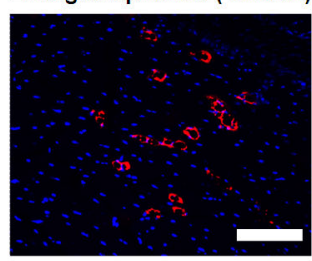

c)
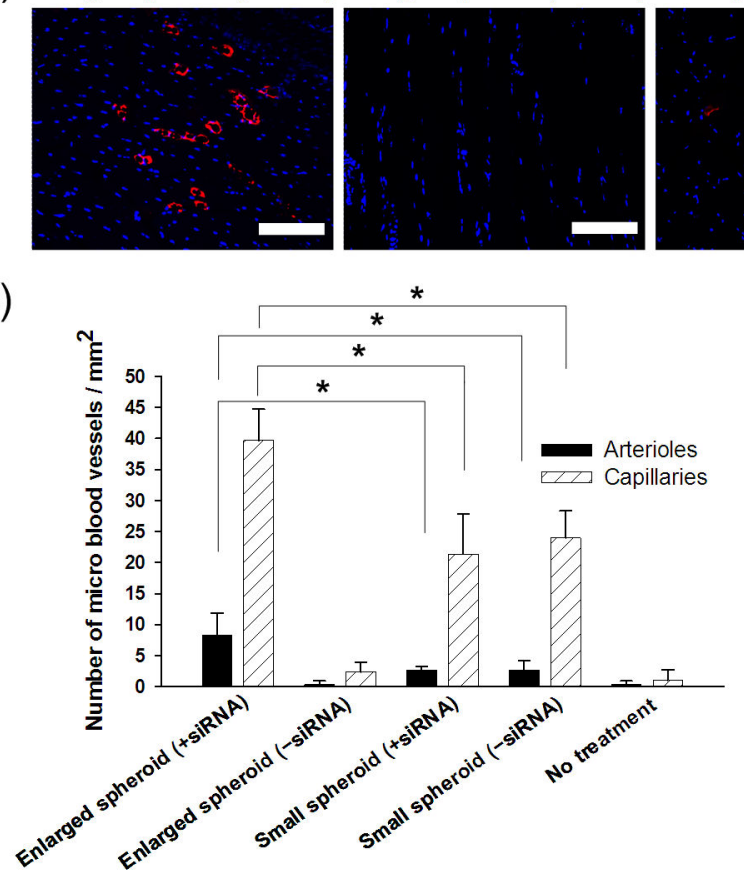
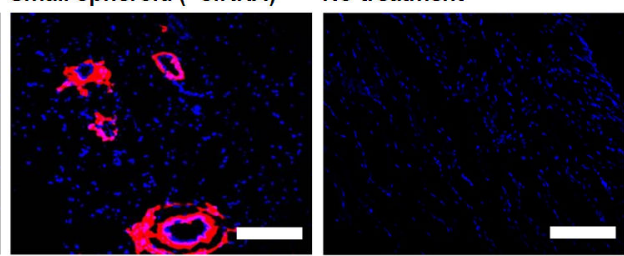

Small spheroid (-siRNA)

No treatment
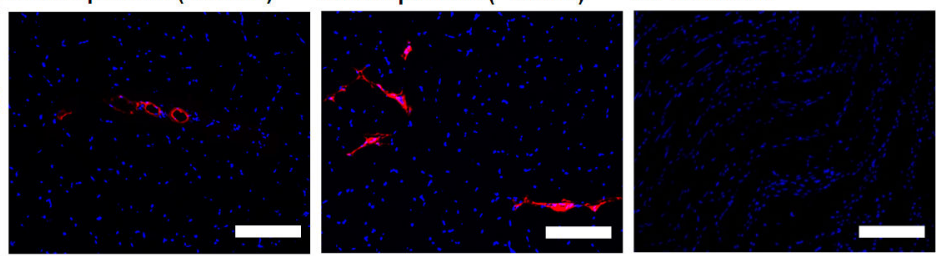

d)

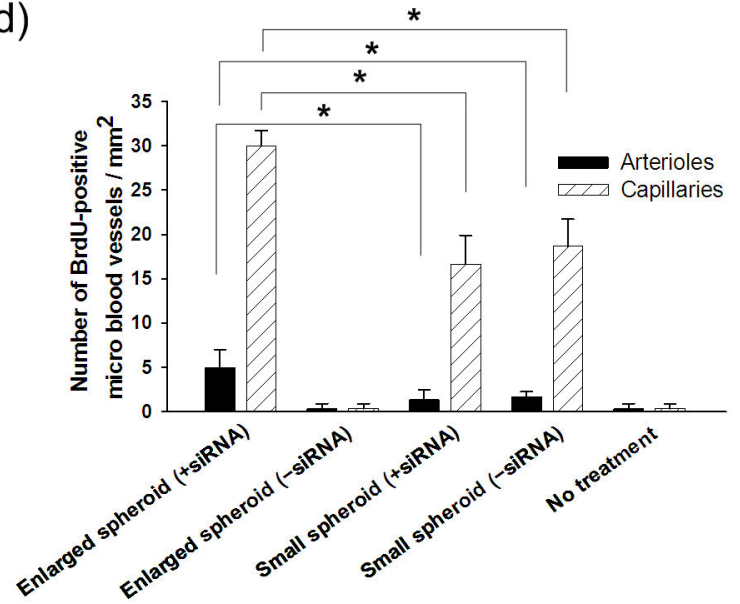

Figure 4.

(a, b) Immunofluorescent staining of (a) SM-a actin (staining of arterioles) and (b) CD31 (staining of capillaries) in the ischemic limb tissues 28 days post transplantation. Nuclei of the cells were counter-stained with DAPI in blue color. The scale bars correspond to 100 $\mu \mathrm{m}$. (c, d) Quantification of (c) total arteriole and capillary density and (d) BrdU-positive arteriole and capillary density in the ischemic regions. * denotes $p<0.05$. 